\title{
TORQUE RIPPLE MINIMISATION IN SWITCHED RELUCTANCE MOTOR WITH OPTIMAL CONTROL OF PHASE CURRENTS
}

\author{
L. Venkatesha, V.Ramanarayanan \\ Department of Electrical Engineering \\ Indian Institute of Science \\ Bangalore INDIA. \\ email : venkat@ee.iisc.ernet.in ; vram@ee.iisc.ernet.in
}

\begin{abstract}
:
This paper presents a method of finding the phase current profiles to get minimum torque ripple in Switched Reluctance Motor (SRM) with maximum torque per ampere. The solutions are obtained with peak current limit, finite rise time due to incremental inductance of the phase windings, and the back emf. As these factors are taken into account in obtaining the solution the implementation do not experience the voltage saturation for all the torque levels up to the rated torque. The solutions are obtained using the flux-linkage characteristics and the static torque characteristics. The influence of speed and the bus voltage on the solutions are discussed. All these results are compared with the results obtained by neglecting the effect of inductance and back emf effect. The implementation of the results are carried out on a $4 \mathrm{KW}, 4$ phase, $8 / 6$ pole OULTON motor using a microcontroller. The simulation and the experimental results are presented.

\section{Introduction:}

The SRM has gained popularity as a variable speed drive in recent years due to several advantages compared to conventional $\mathrm{AC}$ and $\mathrm{DC}$ motors. It has a simple construction with its rotor having no winding or permanent magnet and has high torque to inertia ratio. The constructions and operating principles are well documented $[1,2]$. The primary disadvantage of SRM is the higher torque ripple compared to conventional machines. The torque ripple is objectionable in a high performance servo application and even in other applications, it adds to the vibration and acoustic noise. The origin of higher torque pulsation is due to the non-linear and discrete torque production mechanism. The total torque in SRM is the sum of the torque produced by each of the stator phases. The torque pulsation is significant during the commutation when the torque production shifts from one active phase to the other.
\end{abstract}

There are two main approaches to the problem of reducing the torque ripple. One method is to improve the magnetic design of the motor and the other method is to use appropriate current control: In the first method, machine design is made with special attention to reduce the torque ripple. Some of the published works following the second approach are reviewed here. In the method suggested by J.C. Moreira [3], the instantaneous torque and the rotor position are estimated from terminal voltages and current measurements and it uses the flux-linkage characteristics for determination of coefficients of interpolating polynomial needed to compute the torque. This torque is compared with a constant reference torque and fed to a current regulator for torque control. In this method, computation of the instantaneous torque and rotor position needs fast processing of the data and the reference current generated from this algorithm does not guarantee maximum torque per ampere. In the paper [4], by D.S. Scharamm et al. the phase currents are obtained by widening the commutation period such that torque falls linearly to zero in one phase and rises linearly in the other. In this method, the effect of incremental inductance and the backemf are not taken into account. In [5] a contour function is used for the torque produced by individual phases and the function is chosen such that the resultant torque is constant. The phase currents are computed to get the required torque from static torque characteristics. The solutions obtained by this method do not give maximum torque per ampere and the results shown in the paper neglect saturation.

The proposed method takes into account the incremental inductance of the machine, and the back emf. The solutions are obtained for maximum torque per ampere with peak current 


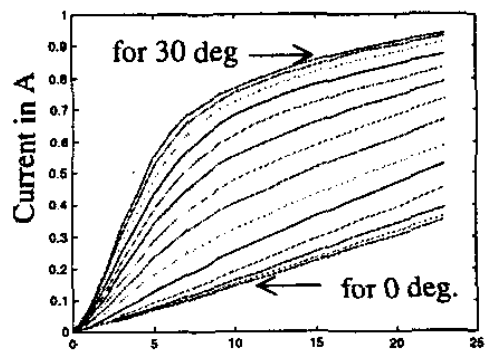

Rotor position in degrees

Fig.1 Flux-linkage characteristics of $4 \mathrm{KW} \mathrm{SRM}$

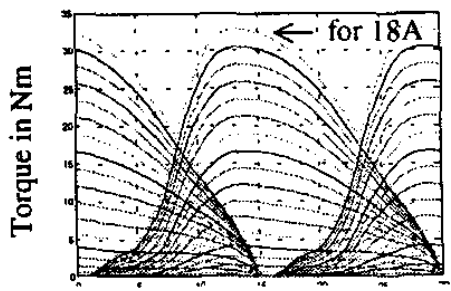

Rotor position in degrees

Fig.2 Static torque characteristics of SRM limit which helps limiting the current stress on the switching devices. The dependence of the solution on the speed and bus voltage are discussed. This method is also applicable for $6 / 8$ pole SRM although the solutions are worked out for $8 / 6$ configuration.

The proposed method:

The proposed method is current trajectory control method. These trajectories are obtained as a solution to obtain zero torque ripple using the static torque characteristics and the flux-linkage characteristics of the machine. The static torque characteristics represent the torque developed versus rotor position with current as parameter. This can be obtained as follows. First the flux-linkage characteristics of the machine are obtained experimentally [6]. Then the static torque characteristics can be obtained by using the following equations at different current levels.

$$
\mathrm{t}=\frac{\partial w^{\prime}}{\partial \theta} l_{\mathrm{i} \text { constant }}
$$

where

$$
w^{\prime}=\int_{0}^{i_{1}} \Psi d i
$$

In the above equations $\mathrm{W}$ ' is the co-energy, ' $\mathrm{i}$ ' is the phase current and $\Psi$ is the flux-linkage.

Static torque can also be obtained by using torque transducer directly. The flux-linkage characteristics and the static torque characteristics for $4 \mathrm{KW}, 8 / 6$ pole SRM are shown in Fig. 1 and Fig. 2 respectively.

\section{Problem Formulation:}

The resultant torque of the motor must be constant for all rotor positions. This can be mathematically expressed as,

$$
t=k_{1} \text { for all } \theta
$$

and $t$ is given by

$$
t=\sum_{n=0}^{n=4} t_{n}
$$

and

$$
t_{n}=\left.\frac{\partial w_{n}}{\partial \theta_{n}}\right|_{i_{n}=\text { constant }}
$$

The solution is sought subjected to the following condition;

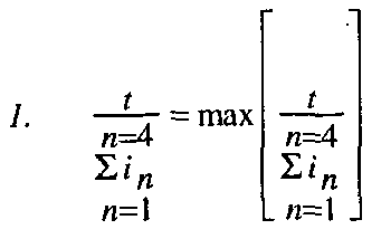

where $i_{n}$ the solution of the equation

$$
\text { 2. } \quad\left[\mathrm{i}_{n}\right]_{\max }=\mathrm{k}_{2}
$$

In the above equations, the constant $k_{1}$ is equal to the desired torque , ' $\mathrm{t}$ ' is the resultant torque, $\mathrm{n}$ is a parameter for phase number, $\mathrm{R}$ is the resistance of the phase winding, $v$ is the applied voltage per phase, $k_{2}$ is a constant and is equal to the peak current limit.

\section{Solution:}

As this problem is quite involved, the solution is worked out using the numerical method and the equations are modified to suit the computation. The following assumptions are made

1. The speed is assumed to remain constant

2. The mutual inductance effect and eddy current effect are neglected.

3. The resistance of the winding is neglected.

To save the computation time following observations are made from the static torque characteristics. For any rotor position, only two 


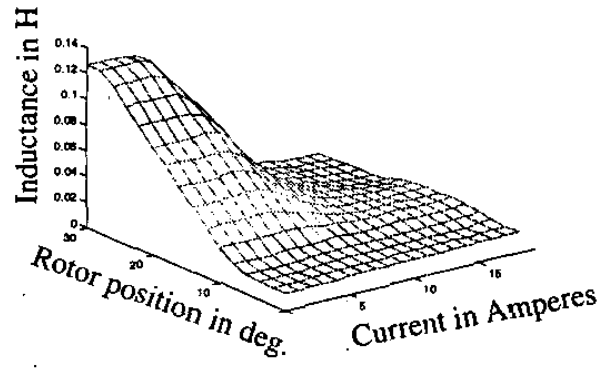

Fig. 3 Surface plot of incremental inductance.

phases can produce positive torque. Among the two phases only one phase will produce the desired torque with minimum current except at one position where the two commutating phases produce the desired torque with same current (at this position torque per ampere values are equal). The change in phase current required has to meet the following constraint which is due to Eq. (7)

$$
\frac{d i_{k}}{d t}=\frac{1}{\frac{\partial \Psi\left(\theta_{k}, i_{k}\right)}{\partial i_{k}}} *\left[v-i_{k} R-\omega \frac{\partial \Psi_{k}}{\partial \theta}\right]
$$

The term $\frac{\partial \Psi}{\partial i}$ corresponds to the incremental inductance and it is obtained by numerically differentiating the flux-linkage characteristics with respect to phase current for any given rotor position. A surface plot of incremental inductance is as shown in Fig. 3. The back emf coefficient $\frac{\partial \Psi}{\partial \theta}$ is obtained by numerically differentiating the flux-linkage with respect to rotor position for any given phase current and these coefficients are multiplied by angular velocity to get the backemf. The surface plot of back emf at $300 \mathrm{rpm}$ is as shown in Fig. 4.

Assuming the voltage per phase to remain constant, the algorithm to obtain the solution is as follows :

1. The solution is obtained for every 0.2 degrees of rotor position.

2 . Time required to cover 0.2 degrees is calculated $(\Delta t)$ for the chosen value of speed

3. For unaligned rotor position of a phase ( say phase 2) the dominating phase (phase 1) is

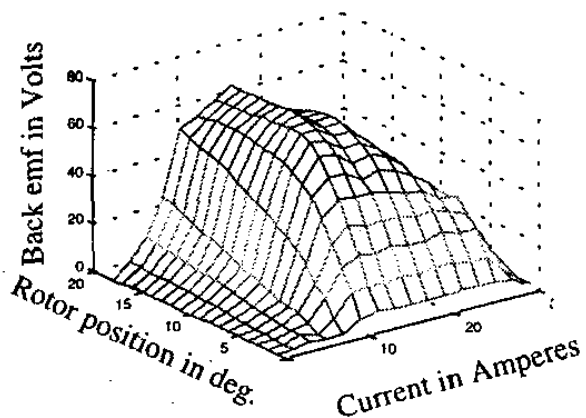

Fig. 4. Surface plot of back emf at $300 \mathrm{rpm}$

allowed to take the desired load torque and to estimate the current required a spline fit is used.

4. For any other position, the maximum and the minimum values on the phase current is calculated using Eq. (9) in difference form as follows

$$
\begin{aligned}
i_{1 \max }\left(\theta_{n}\right)= & i_{1}\left(\theta_{n-1}\right) \\
& +\frac{v-e_{b 1}\left(\theta_{n-1}, i_{1}\left(\theta_{n-1}\right)\right)}{l\left[\theta_{n-1}, i_{1}\left(\theta_{n-1}\right)\right]} \Delta t \\
i_{1 \min }\left(\theta_{n}\right)= & i_{1}\left(\theta_{n-1}\right) \\
& -\frac{v-e_{b 1}\left(\theta_{n-1}, i_{1}\left(\theta_{n-1}\right)\right)}{l\left[\theta_{n-1}, i_{1}\left(\theta_{n-1}\right)\right]} \Delta t \\
i_{2 \max }\left(\theta_{n}\right)= & i_{2}\left(\theta_{n-1}\right) \\
& +\frac{v-e_{b 2}\left(\theta_{n-1}, i_{2}\left(\theta_{n-1}\right)\right)}{l\left[\theta_{n-1}, i_{2}\left(\theta_{n-1}\right)\right]} \Delta t \\
i_{2 \min }\left(\theta_{n}\right)= & i_{2}\left(\theta_{n-1}\right) \\
& -\frac{v-e_{b 2}\left(\theta_{n-1}, i_{2}\left(\theta_{n-1}\right)\right)}{l\left[\theta_{n-1}, i_{2}\left(\theta_{n-1}\right)\right]} \Delta t
\end{aligned}
$$

The maximum current of the phases are limited to peak current limit and minimum current to zero.

5. At each rotor position, the phase in which the required torque can be obtained with minimum current is allowed to take the entire torque if the computed current is within the current limits as obtained from Eq. 10-13. If the phase current reaches the peak current limit and if the torque produced is less than the desired torque, then the next positive torque producing phase is allowed to take the remaining torque. When the domination changes from the first to the second phase, the second phase current is allowed to rise up to a value which is governed by its maximum current limit, the restriction that arises from the rate of 
fall of current in the other phase and the constant resultant torque condition.

6. This procedure is repeated until the unaligned position of the next phase (phase 3) is reached. At this position the solution repeats if the current in the outgoing phase (phase 1) has reached zero. If not, the dominant torque producing phase (phase 2) is called upon to compensate for the negative torque produced by the outgoing phase. The corrected results are used as reference for all the phases.

The computed results for phase voltage of $220 \mathrm{~V}$ and a speed of $150 \mathrm{rpm}$ are shown in Fig. 5. A peak current limit of $18 \mathrm{~A}$ is used to obtain the solution.

Influence of bus voltage and the speed

As the speed changes the back emf and the time required to cover the angle step considered for solution changes. Therefore the upper and lower limit for the current changes as a function of speed. The computed results for a bus voltage of $440 \mathrm{~V}$. and a speed of $300 \mathrm{rpm}$ are shown in Fig. 6 . The results show that the current in the phase will not go to zero before 30 degrees as in the case of $150 \mathrm{rpm}$. Therefore the computations are continued till the solution repeats.

The solutions for the current trajectories are computed for a bus voltage of $560 \mathrm{~V}$ to understand the influence of the bus voltage. The upper and lower permissible limit for the currents will increase with increase in the bus voltage for a given speed. The computation is carried out for $150 \mathrm{rpm}$ and $560 \mathrm{~V}$ and the results are used in comparison.

\section{Comparison of computed results}

The results obtained for $150 \mathrm{rpm}$ and $300 \mathrm{rpm}$ are compared with that of the case where the current can be instantaneously increased to the desired level. This comparison is made to understand the influence of back-emf and incremental inductance of the machine which restrict the rate of rise of current in the phase once that phase takes the dominance. The comparison results is shown in Fig. 7. It is observed that the conduction angle increases with speed The average phase current also increases with the speed for a given torque.

Figure 8 shows the comparison of results for 150 rpm with DC bus voltages of $440 \mathrm{~V}$ and $560 \mathrm{~V}$. The current trajectories change as the rate of rise of current in the incoming phase and fall of current in the outgoing phase reduces at $440 \mathrm{~V}$ level. There is no appreciable change in the average phase current, when the bus voltage is changed by about $20 \%$.

\section{Experimental results :}

The implementation of the above scheme is carried out on a $4 \mathrm{KW}, 8 / 6$ pole OULTON SRM using a microcontroller (80196KC). The computed current values are stored in a look up table. The program is interrupt driven. A software timer interrupt occurs for every 0.2 degrees of rotation of the rotor. When the interrupt occurs the reference currents for one or two phases (depending on the rotor position) are generated using the lookup table. Linear interpolation is used for intermediate torque levels. The rotor position information is obtained from the rotor position sensors. The reference currents are the compared with the actual currents to generate the switching pulses. These pulses are fed to the frequency limiting circuit and then fed to the converter. The result obtained for $150 \mathrm{rpm}$ with a $\mathrm{DC}$ bus voltage of $440 \mathrm{~V}$ is shown in Fig 9. The figure shows the torque developed due to individual phases and the resultant torque. The torque waveform is obtained from the rotor position and the current waveforms.

Influence of switching frequency:

The torque ripple can be eliminated if the computed currents are established in the phases at the required rotor positions. The experimental result shows a torque ripple of about $18 \%$ [The experiments are carried out with a maximum frequency limit of $8 \mathrm{Khz}$.$] . As these reference$ currents are established by means of chopping, there will be current ripple. The current ripple results in the torque ripple. The current ripple and hence the torque ripple can be reduced further by using higher switching frequency. Simulation is carried out to observe the influence of higher switching frequency on the torque ripple and results are shown with switching frequency limit of $40 \mathrm{Khz}$ in Fig. 10. The maximum torque ripple is less than $3 \%$.

\section{Conclusion}

In this paper, a method to compute the phase currents for torque ripple minimisation in SRM has been described. The solutions are obtained numerically to get maximum torque per ampere and with peak current limit. The back emf and 


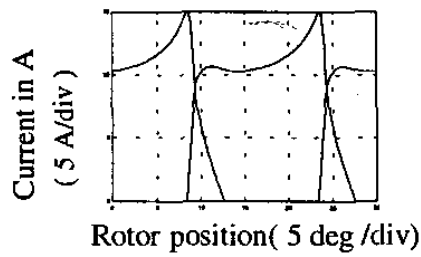

( a)

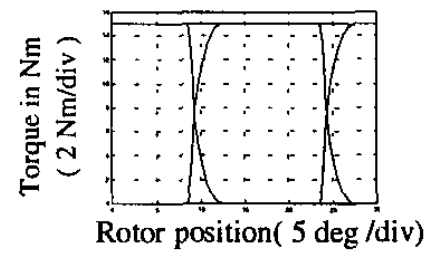

( b)

a. Phase current vs rotor position

b Torque vs rotor position

Fig. 5 The computed phase currents at $150 \mathrm{rpm}$ with a bus voltage of $440 \mathrm{~V}$

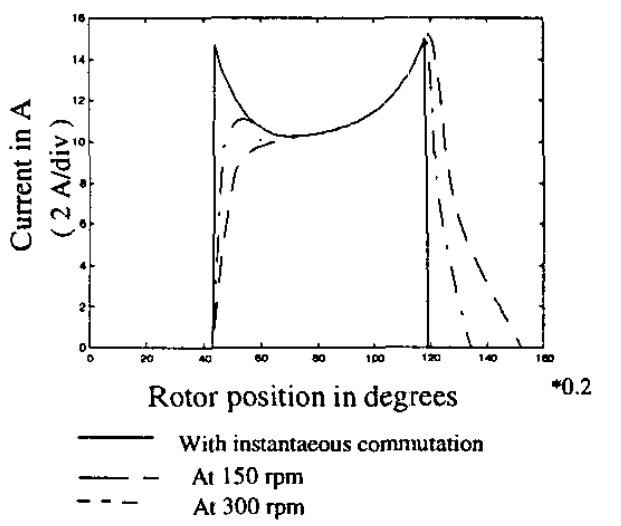

Fig. 7 Comparison of computed currents for 150 and $300 \mathrm{rpm}$ with a bus voltage of $560 \mathrm{~V}$

incremental inductance effects are considered in obtaining the solution. The solution results in an increase in the $\mathrm{Nm} / \mathrm{A}$ rating of the inverter without exceeding the peak current stress on the devices. The solutions are worked out for two different bus voltages and speeds. It is observed that the conduction angle increases with speed. The peak current limit increases the commutation zone and the conduction angle. When the bus voltage is changed by about $20 \%$, the average
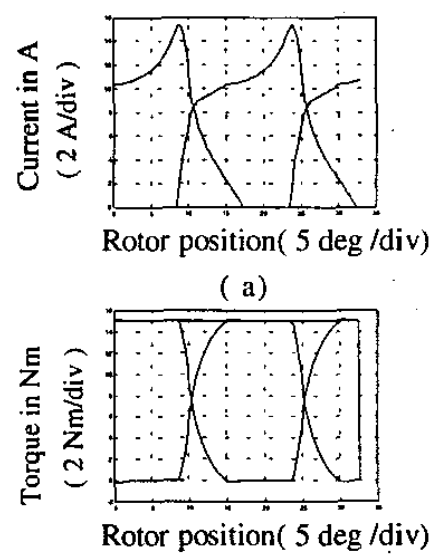

(b)

a. Phase current vs rotor position

b Torque vs rotor position

Fig. 6 The computed phase currents at $300 \mathrm{rpm}$ with a bus voltage of $440 \mathrm{~V}$

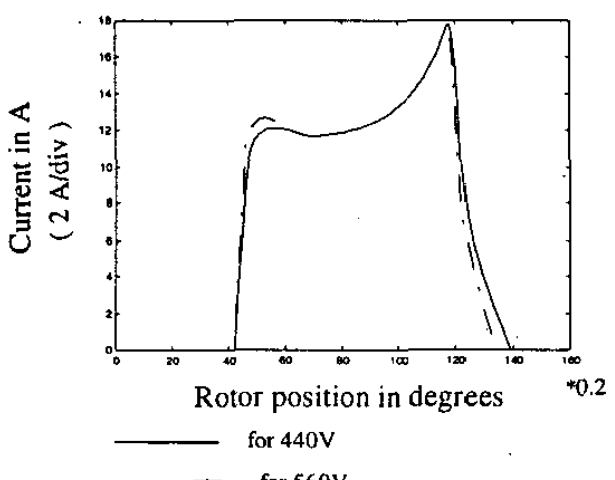

Fig. 8 Comparison of computed currents for 440 and $560 \mathrm{rpm}$ with a bus voltage . of $150 \mathrm{rpm}$

current does not appreciably differ. The implementation results show that the torque ripple is appreciably less compared to the conventional constant current reference control scheme. Simulation shows that the maximum torque ripple can be further reduced below $3 \%$ with a switching frequency limit of $40 \mathrm{Khz}$. 

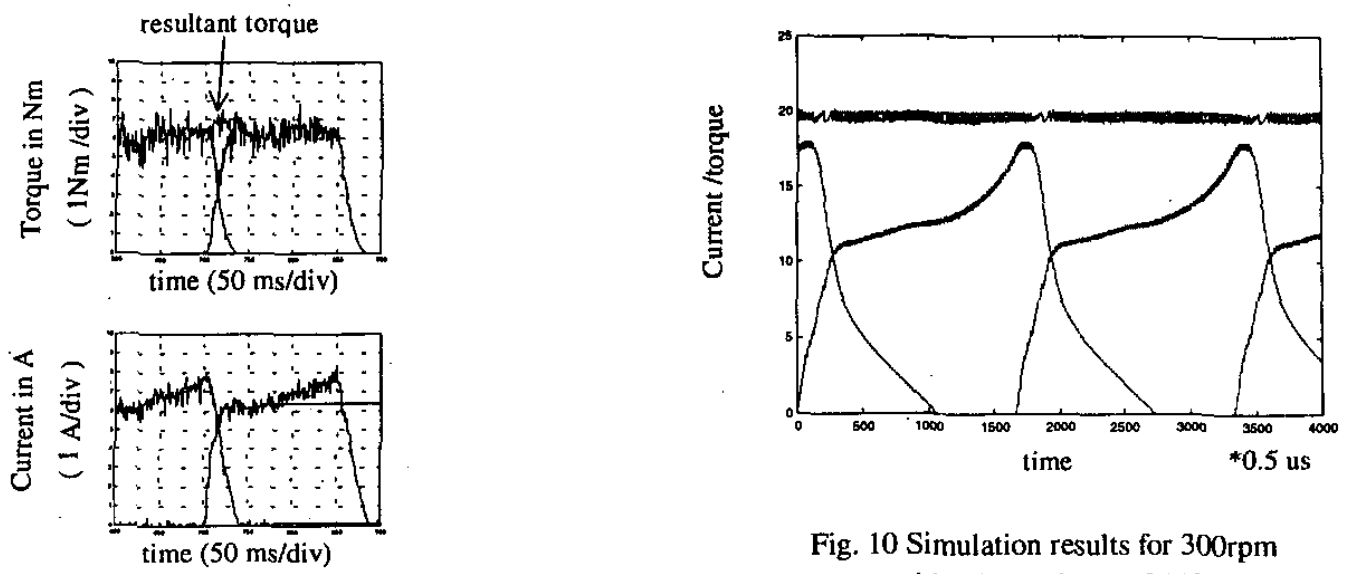

Fig 9 Experimental results illustrating the commutation zone at $150 \mathrm{rpm}$ with a bus voltage of $440 \mathrm{~V}$

\section{References:}

1. P.J. Lawrenson, J.M.Stephenson, P.T Blenkinsop, J.Corda and N.N. Fulton, "Variable speed reluctance motors " IEE Proc. Pt. B, Vol; 127, no. 4, July 1980 .

2. T.J.E Miller "Switched Reluctance Motors and their Control" Magna Physics publishing and Clarendon Oxford 1993.

3. J. C. Moreira " Torque ripple minimisation in switched reluctance motor via bi-cubic spline interpolation," IEEE PESC Conf, Rec. 1992.

4. D.S. Schramm, B.W. Williams and T.C. Green, "Torque ripple reduction of Switched reluctance motors by phase current optimal profiling, " IEEE-PESC Conf. Rec.1992

5. Iqbal Husain, Mehardad Eshani,"Torque ripple minimisation in Switched reluctance motor drives by PWM current control," IEEE-PESC Conf. Rec.1994.

6. V. Ramanarayanan, L.Venkatesha, D.P.Panda," Flux-linkage Characteristics of Switched Reluctance Motor," PEDES '96 Conf. Rec. 1996. 